\title{
Root Identifier
}

National Cancer Institute

\section{Source}

National Cancer Institute. Root Identifier. NCI Thesaurus. Code C70903.

A unique identifier that guarantees the global uniqueness of the instance identifier. The root alone may be the entire instance identifier. 